NISSUNA UMANA INVESTIGAZIONE SI PUO DIMANDARE VERA SCIENZIA S'ESSA NON PASSA PER LE MATEMATICHE DIMOSTRAZIONI LEONARDO DA VINCI

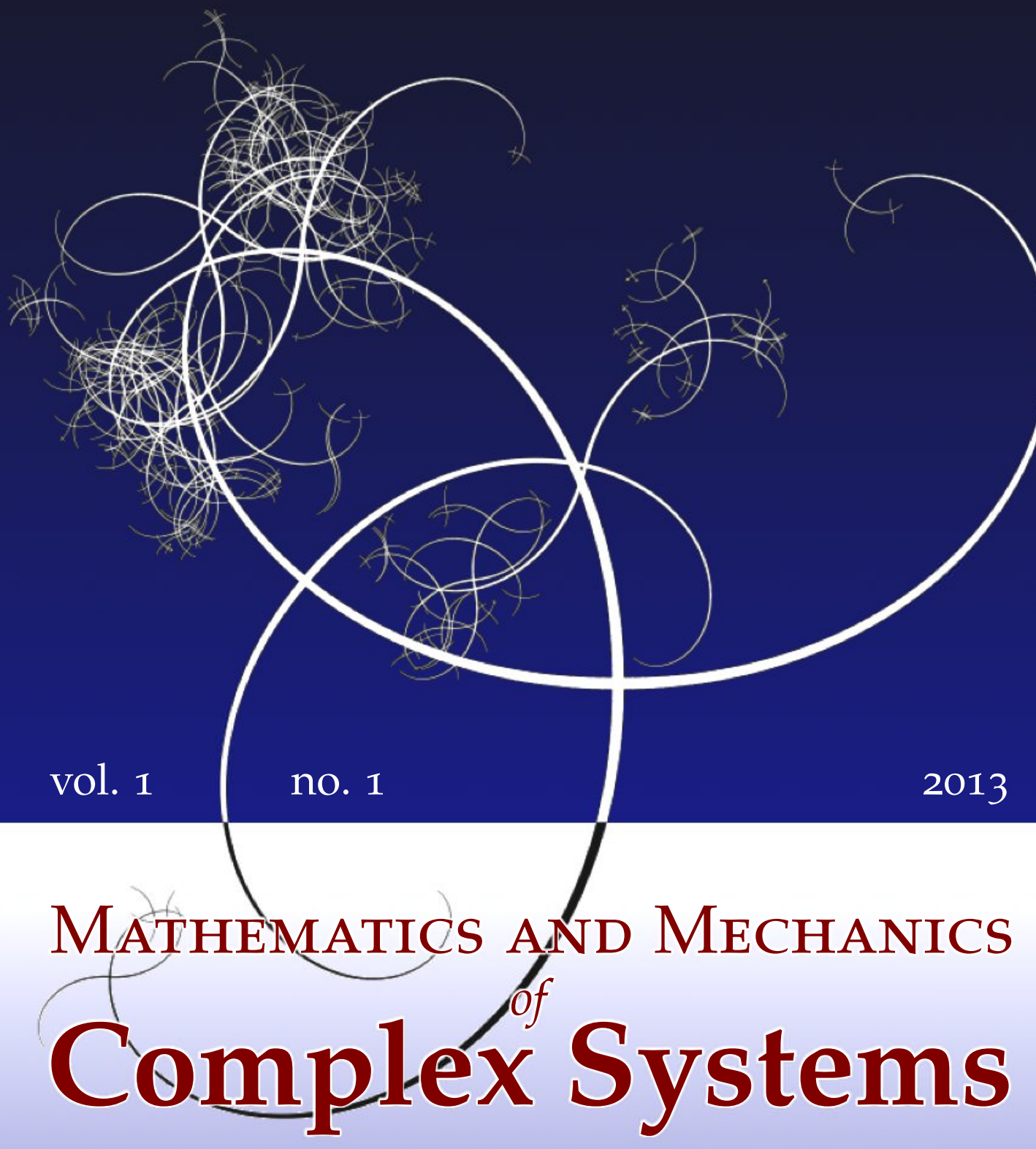

Gary J. Templet and David J. Steigmann

ON THE THEORY OF DIFFUSION AND SWELLING IN FINITELY DEFORMING ELASTOMERS 


\title{
ON THE THEORY OF DIFFUSION AND SWELLING IN FINITELY DEFORMING ELASTOMERS
}

\author{
GARY J. TEMPlet AND DAVid J. STEIGMANN
}

\begin{abstract}
The role of a relaxed local intermediate configuration associated with free swelling is examined in the context of diffusion of a liquid in an isotropic elastomer. It is found that this configuration is energetically optimal if the free-energy function of the polymer-liquid gel is polyconvex. Further aspects of the general theory of diffusion in elastomers are also discussed.
\end{abstract}

\section{Introduction}

We study the modern continuum theory for diffusion of an incompressible liquid in an incompressible elastomer [Treloar 1975; Truesdell 1962; Adkins 1964; Weitsman 1987; Shi et al. 1981; Rajagopal 2003; Baek and Srinivasa 2004; Prasad and Rajagopal 2006]. In addition to examining the structure of constitutive equations and initial-boundary-value problems, we study the role of a local intermediate configuration induced by free swelling, defined as dilation of the polymer network in the absence of stress due to the presence of an infused liquid. We show, for isotropic elastomers, that the local free-swelling deformation minimizes energy if the freeenergy function for the polymer network satisfies the condition of polyconvexity. In fact we are able to weaken this requirement to rank-one convexity. Accordingly the decomposition of the deformation into elastic and swelling deformations, assumed a priori in current theories of diffusion in polymers [Pence and Tsai 2005; Hong et al. 2008; Duda et al. 2010; Chester and Anand 2011; Duda et al. 2011], is meaningful for free-energy functions commonly used to model elastomers. Moreover, because of the basic role played by polyconvexity in Ball's landmark existence theory [1977] for conventional elasticity, our results indicate that an extension of that theory to accommodate diffusion should be feasible.

We use standard notation such as $\boldsymbol{A}^{t}, \boldsymbol{A}^{-1}, \boldsymbol{A}^{*}, \operatorname{tr} \boldsymbol{A}$ and $J_{A}$. These are respectively the transpose, the inverse, the cofactor, the trace and the determinant of a tensor $\boldsymbol{A}$, regarded as a linear transformation from a three-dimensional vector space to itself, the latter being identified with the translation space of the usual three-dimensional Euclidean point space. We also use $\mathrm{Sym}^{+}$to denote the linear

\section{Communicated by Pierre Seppecher.}

MSC2010: 74FXX.

Keywords: diffusion, polymers, polyconvexity. 
space of positive symmetric tensors and $\mathrm{Orth}^{+}$to denote the group of rotation tensors. We denote the standard tensor product of vectors by interposing the symbol $\otimes$. The Euclidean inner product of tensors $\boldsymbol{A}$ and $\boldsymbol{B}$ is $\boldsymbol{A} \cdot \boldsymbol{B}=\operatorname{tr}\left(\boldsymbol{A} \boldsymbol{B}^{t}\right)$, and the associated norm is $|\boldsymbol{A}|=\sqrt{\boldsymbol{A} \cdot \boldsymbol{A}}$. Lastly, $D$ is used to denote the gradient with respect to position $\boldsymbol{x}$ in a reference configuration and $F_{\boldsymbol{A}}$ stands for the tensorvalued derivative of a scalar-valued function $F(\boldsymbol{A})$.

Section 2 contains an outline of the general theory, including the diffusive balance law, the swelling constraint and the definition of dissipation. The latter is shown to lead to a constitutive structure in which the stress and chemical potential are determined, modulo a Lagrange multiplier, by a free-energy function that depends on the deformation gradient and the concentration of diffusant. The role played by polyconvexity in this theory is examined and used to analyze the freeswelling problem, which forms the basis of a decomposition of the deformation into elastic and swelling components. The general constitutive equation for the mobility tensor is also examined and shown to satisfy all invariance and symmetry requirements. The relatively simple theory for two-dimensional problems is summarized in Section 3, and an Appendix is included in which necessary and sufficient conditions for polyconvexity in respect of isotropic materials are discussed.

\section{Three-dimensional theory}

2.1. Basic equations and inequalities. We outline the basic theory for diffusion in elastomers undergoing finite deformations. For the most part our model may be viewed as a specialization of a thermodynamical theory presented in [Gurtin et al. 2010]. Here, however, thermal effects are suppressed, and the model reverts to conventional finite-elasticity theory in the absence of diffusion. The effects of inertia are also suppressed, in deference to the fact that the associated time scales typically differ markedly from those associated with the effects of diffusion. Further, we do not take chemical reactions into account, although it is possible to do so in a manner that is compatible with the present framework.

Let $c(\boldsymbol{x}, t)$ be the concentration of diffusant, where $t$ is the time and $\boldsymbol{x}$ is position in a fixed reference placement $\kappa$. For example, it is common to take the concentration to be the number of molecules of diffusant per unit volume of the reference configuration adopted for the dry elastomer [Chester and Anand 2011], the latter then being identified with $\kappa$. Alternatively, in [Gurtin et al. 2010] the concentration is defined to be the mass of diffusant per unit reference volume. The ratio of the second of these definitions to the first is the mass of a molecule of diffusant, a constant. Accordingly, these definitions are equivalent. A third definition of $c$, equivalent to these and more convenient for our purposes, is introduced in the next subsection. 
For any subvolume $\pi$ of $\kappa$ we have the diffusive balance

$$
\frac{d}{d t} \int_{\pi} c d v=-\int_{\partial \pi} \boldsymbol{m} \cdot \boldsymbol{n} d a \text { for all } \pi \subset \kappa,
$$

where $\boldsymbol{m}(\boldsymbol{x}, t)$ is the flux of diffusant and $\partial \pi$ is the piecewise smooth boundary, with exterior unit normal $\boldsymbol{n}$, of the region $\pi \subset \kappa$. The inequality $\boldsymbol{m} \cdot \boldsymbol{n}>0$ (resp., $<0$ ) corresponds to local transport of diffusant out of (resp., into) $\pi$; transport vanishes when $\boldsymbol{m} \cdot \boldsymbol{n}$ vanishes. The flux $\boldsymbol{m}$ arises entirely from diffusant transport, resulting in a change of mass of the gel (polymer-diffusant mixture) associated with $\kappa$. Consistent with this interpretation, we suppress bulk production of diffusant, which would otherwise require the addition of a volumetric source term to (1).

The local form of (1) is

$$
\dot{c}+\operatorname{Div} \boldsymbol{m}=0 \quad \text { in } \kappa,
$$

where the superposed dot stands for $\partial / \partial t$ at fixed $\boldsymbol{x}$ and Div is the divergence with respect to $\boldsymbol{x}$.

We assume the existence of a free-energy function $\Psi(\boldsymbol{F}, c)$, yielding the energy of the gel per unit volume of $\kappa$, where $\boldsymbol{F}$, with $J_{F}>0$, is the gradient of a deformation function $\boldsymbol{y}=\chi(\boldsymbol{x}, t)$ yielding the position at time $t$ of a point associated with position $\boldsymbol{x} \in \kappa$. Thus $\chi$ represents the motion of the infused elastomer. For convenience we suppress reference to a possible explicit dependence on $\boldsymbol{x}$ in the notation for the free-energy function.

The power supplied to the arbitrary part $\pi$ of the body, assuming no body forces and no volumetric sources of diffusant, is

$$
P(\pi, t)=\int_{\partial \pi}(\boldsymbol{p} \cdot \dot{\boldsymbol{y}}-\boldsymbol{q} \cdot \boldsymbol{n}) d a,
$$

where

$$
\boldsymbol{p}=\boldsymbol{P n},
$$

in which $\boldsymbol{P}$ is the Piola stress, is the traction acting on the boundary, and $\boldsymbol{q}$ is the rate of energy supply due to the flux of diffusant; that is, the rate at which the energy content of the gel changes due to the transport of diffusant across $\partial \pi$. The sign appearing before $\boldsymbol{q}$ conforms to the convention adopted in (1). With this interpretation of $\boldsymbol{q}$, it follows on logical grounds alone that

$$
\boldsymbol{q}=\mu \boldsymbol{m},
$$

where $\mu$, the chemical potential, is the energy conducted by the diffusant into the gel; ie., the energy per unit of diffusant concentration. The same conclusion follows from a rigorous thermodynamic treatment of diffusion in which the chemical potential is admitted as an independent variable [Gurtin et al. 2010]. 
Following [Gurtin et al. 2010] we suppose that $\boldsymbol{m}$ depends constitutively on the list $\{\boldsymbol{F}, c, D \mu\}$, while $\boldsymbol{P}$ depends constitutively on $\{\boldsymbol{F}, c\}$. Further, if the net force on an arbitrary subvolume $\pi \subset \kappa$ vanishes, as we assume, then the stress satisfies

$$
\operatorname{Div} \boldsymbol{P}=\mathbf{0} \text { in } \kappa \text {. }
$$

The dissipation is denoted by $\mathscr{D}(\pi, t)$ and defined as the difference between the power supplied to $\pi$ and the rate of change of the total energy in $\pi$. Thus,

$$
\mathscr{D}(\pi, t)=P(\pi, t)-\frac{d}{d t} \int_{\pi} \Psi d v .
$$

It is assumed to be non-negative for every subregion; i.e.,

$$
\mathscr{D}(\pi, t) \geq 0 .
$$

Using (4), applying the divergence theorem and supposing all fields to be smooth in $\pi$ yields

$$
\int_{\partial \pi} \boldsymbol{p} \cdot \dot{\boldsymbol{y}} d a=\int_{\pi} \operatorname{Div}\left(\boldsymbol{P}^{t} \dot{\boldsymbol{y}}\right) d v
$$

in which

$$
\operatorname{Div}\left(\boldsymbol{P}^{t} \dot{\boldsymbol{y}}\right)=\boldsymbol{P} \cdot \dot{\boldsymbol{F}},
$$

by virtue of (4) and (6). Accordingly, (2), (3), (7) and (10), together with $\operatorname{Div}(\mu \boldsymbol{m})=$ $\mu$ Div $\boldsymbol{m}+\boldsymbol{m} \cdot D \mu$, furnish

$$
\mathscr{D}(\pi, t)=\int_{\pi}(\boldsymbol{P} \cdot \dot{\boldsymbol{F}}+\mu \dot{c}-\dot{\Psi}-\boldsymbol{m} \cdot D \mu) d v,
$$

and (8) then yields the local restriction

$$
\left(\Psi_{\boldsymbol{F}}-\boldsymbol{P}\right) \cdot \dot{\boldsymbol{F}}+\left(\Psi_{c}-\mu\right) \dot{c}+\boldsymbol{m} \cdot D \mu \leq 0 .
$$

\subsection{Volume decomposition, the swelling constraint and the basic constitutive} structure. Most workers adopt the assumption that the volume of the gel is simply the sum of the volumes of the elastomer and the liquid diffusant. We invoke this assumption here and thus conclude that for any $\pi \subset \kappa$,

$$
\int_{\pi} J_{F} d v=\int_{\pi}\left(J_{F e}+J_{F d}\right) d v,
$$

where $J_{F e}$ and $J_{F d}$ respectively are the current volume of elastomer and diffusant per unit reference volume. Accordingly,

$$
J_{F}=J_{F e}+J_{F d} \quad \text { in } \quad \kappa .
$$

Here and henceforth we assume that sufficient liquid is available to support dilation. The alternative, corresponding to an unsaturated condition [Deng and Pence 2010; 
Rivlin 1977], entails the global constraint that the total dilation reduce to the sum of the elastomer and liquid volumes. This carries a uniform reaction pressure that must be added to the expression for the (Cauchy) stress (see previous references).

Following [Chester and Anand 2011], here we suppose the contribution of the diffusant to arise solely from its transport into $\kappa$. Thus,

$$
J_{F d}=c_{d} v_{d},
$$

where $c_{d}$ is the number of molecules of diffusant per unit volume of $\kappa$ and $v_{d}$ is the volume of a molecule of diffusant. The latter is presumed to be constant if the diffusant is incompressible. Accordingly, $J_{F d}$ and $c_{d}$ furnish equivalent definitions of concentration in this case. The assumption that the elastomer is essentially incompressible is also virtually ubiquitous. This implies that $J_{F e}=1$ and hence the swelling constraint [Chester and Anand 2011]

$$
J_{F}=1+c, \quad \text { where } c=c_{d} v_{d},
$$

applicable to incompressible elastomers infused with an incompressible diffusant. Because $c$ is defined as the product of non-negative scalars, it follows from (16) that

$$
J_{F} \geq 1 \text {. }
$$

The simplicity of (16) justifies our choice of $c$ - the volume of diffusant present in the gel per unit volume of dry elastomer - as the measure of concentration. Accordingly, for a gel consisting of an incompressible elastomer infused with an incompressible diffusant, the deformation and concentration are not independent.

To account for this interdependence in (12), we use (16) in the form $\dot{c}=\dot{J}_{F}$, together with $\dot{J}_{F}=\boldsymbol{F}^{*} \cdot \dot{\boldsymbol{F}}$, obtaining

$$
\left[\Psi_{\boldsymbol{F}}-\boldsymbol{P}+\left(\Psi_{c}-\mu\right) \boldsymbol{F}^{*}\right] \cdot \dot{\boldsymbol{F}}+\boldsymbol{m} \cdot D \mu \leq 0,
$$

in which $\dot{\boldsymbol{F}}$ is unrestricted and, importantly, $\boldsymbol{F}$ and $c$ are to be regarded as being independent when computing the derivatives of $\Psi$. Thus $\Psi$ is regarded as a smooth extension of the free-energy function from the nine-dimensional manifold in $\mathbb{R}^{10}$ defined by the constraint (16). The extended function is thus defined and differentiable for all $\{\boldsymbol{F}, c\} \in \mathbb{R}^{10}$. Accordingly, our constitutive hypotheses may be combined with a standard argument (e.g. [Liu 2002]) to conclude from (18) that

$$
\boldsymbol{P}=\Psi_{\boldsymbol{F}}-q \boldsymbol{F}^{*}, \quad \text { where } q=\mu-\Psi_{c},
$$

together with

$$
\boldsymbol{m} \cdot D \mu \leq 0 .
$$

Because the argument is purely local in space and time, $q$ may vary with $\boldsymbol{x}$ and $t$. Accordingly, it is an additional field to be determined in the course of the analysis 
of the initial-boundary-value problem, which includes the constraint (16). The dissipation in any subvolume is then given by

$$
\mathscr{D}(\pi, t)=-\int_{\pi} \boldsymbol{m} \cdot D \mu d v
$$

and satisfies inequality (8). The connection $\boldsymbol{P}=\boldsymbol{T} \boldsymbol{F}^{*}$, where $\boldsymbol{T}$ is the Cauchy stress, implies that $q$ is mechanically indistinguishable from a pressure. Equivalent results are derived in [Chester and Anand 2011], albeit by a rather circuitous procedure.

Our hypotheses, together with an important result due to Gurtin [2000], yield the general constitutive structure compatible with (20) in the form

$$
\boldsymbol{m}=\boldsymbol{M}(\boldsymbol{F}, c, D \mu) D \mu,
$$

in which the (2nd order) mobility tensor $\boldsymbol{M}$ satisfies

$$
D \mu \cdot \boldsymbol{M}(\boldsymbol{F}, c, D \mu) D \mu \leq 0 .
$$

In this work we restrict attention to the practically important case in which the mobility tensor is insensitive to $D \mu$. Its symmetric part is therefore non-positive, and is negative definite if (23) holds as a strict inequality for all non-zero $D \mu$, so that $\boldsymbol{m} \cdot D \mu=0$ if and only if $D \mu=\mathbf{0}$. Henceforth we assume this to be the case.

Thus the problem is to solve the five equations consisting of the diffusive balance (2), the equilibrium equation (6), and the swelling constraint (16) for the five variables in the list $\{\chi, c, q\}$. Standard boundary data entail the specification of $\boldsymbol{p}$ or $\boldsymbol{y}$, and $\mu$ or $\boldsymbol{m} \cdot \boldsymbol{n}$, on (possibly different) complementary parts of $\partial \kappa$. A distribution of chemical potential may be specified in the domain $\kappa$ at an initial time, and the constitutive equation $(19)_{2}$ may be used to generate an initial distribution of concentration in terms of the specified chemical potential field and the (unknown) initial deformation gradient and Lagrange multiplier fields; the swelling constraint, the equilibrium equation and associated boundary data may then be used to determine initial deformation and Lagrange multiplier fields, and the diffusive balance (2) then used to obtain the initial distribution of the time derivative of concentration. This information is used to advance the concentration in time, and the procedure then repeated for a specified time interval to generate the evolving spatial distribution of the list $\{\chi, c, q\}$.

Frame invariance of material response requires that the free-energy function and the mobility tensor depend on $\boldsymbol{F}$ via the right Cauchy-Green deformation tensor, or equivalently via the right stretch tensor [Gurtin et al. 2010]. The restriction on the free-energy function is equivalent to the symmetry of the Cauchy stress [Rajagopal and Srinivasa 2012] and so renders the moment-of-momentum balance redundant whenever the linear momentum balance is satisfied. 
We apply (20) for $\pi=\kappa$ and suppose traction $\boldsymbol{p}$ to be assigned on a part of the boundary and position to be assigned on the complementary part. Suppose the diffusant flux $\boldsymbol{m} \cdot \boldsymbol{n}$ and chemical potential $\mu$ vanish on complementary parts of $\partial \kappa$. If the traction field is conservative in the sense that the power is expressible as

$$
P(\kappa, t)=\frac{d}{d t} L,
$$

where $L$ is a suitable load potential, then

$$
\frac{d}{d t} E(\kappa, t)+\mathscr{D}(\kappa, t)=0
$$

where $\mathscr{D}(\kappa, t)$ is given by (21) with $\pi=\kappa$, and

$$
E=\int_{\kappa} \Psi d v-L
$$

is the total potential energy of the gel. Inequality (8) then implies that the potential energy is dissipated.

Specifically, the energy associated with the state $\{\boldsymbol{\chi}(\boldsymbol{x}, t), c(\boldsymbol{x}, t)\}$ is no larger than that associated with state $\{\chi(x, t-\tau), c(x, t-\tau)\}$ for any $\tau>0$. Therefore the energy of a state at fixed time $t$ is optimal relative to any trajectory tending to that state. Consequently, if $\{\boldsymbol{\chi}(\boldsymbol{x}, t), c(\boldsymbol{x}, t)\}$ is a trajectory starting at $\left\{\chi\left(\boldsymbol{x}, t_{0}\right), c\left(\boldsymbol{x}, t_{0}\right)\right\}$ and tending to $\left\{\chi_{\infty}(\boldsymbol{x}), c_{\infty}(\boldsymbol{x})\right\}$ a.e. as $t \rightarrow \infty$, then $E_{\infty} \leq E_{0}$, where $E_{0}$ and $E_{\infty}$ are the values of $E$ at times $t_{0}$ and infinity; asymptotically stable states thus minimize the potential energy. Moreover, if the decay of energy is gradual, in the sense that $E(t-\tau) \rightarrow E(t)+o(\tau)$ as $t \rightarrow \infty$, then $d E / d t \rightarrow 0$ as $t \rightarrow \infty$. In this case we have from (21) that $D \mu_{\infty}(\boldsymbol{x})=\mathbf{0}$, where $\mu_{\infty}$ is the large-time limit of the chemical potential. Indeed, the alternative implies, via (23), that there is some $\overline{\boldsymbol{x}} \in \kappa$ where $D \mu_{\infty} \cdot \boldsymbol{M}\left(\boldsymbol{F}_{\infty}, \sigma_{\infty}\right) D \mu_{\infty}<0$. By continuity, the latter inequality obtains in a subvolume of non-zero measure, and a contradiction then follows from the fact that $\mathscr{D} \rightarrow 0$ as $t \rightarrow \infty$.

In practice the diffusive balance (2) is integrated in time using Euler forward differencing, for example, to generate the concentration $c\left(\boldsymbol{x}, t_{n}\right)$ at time $t_{n}$, say. One then seeks a deformation $\chi\left(\boldsymbol{x}, t_{n}\right)$ that minimizes the energy under the constraint $J_{F}\left(\boldsymbol{x}, t_{n}\right)=1+c\left(\boldsymbol{x}, t_{n}\right)$. This procedure motivates a definition, in Section 2.4, of a kinematic decomposition of the deformation gradient into elastic and swelling parts in which the latter is associated with an unstressed state at an assigned value of $c$. It also suggests a framework whereby an extension of Ball's theory [1977], in which polyconvexity figures prominently, may be explored to establish the existence of an energy minimizing deformation $\chi\left(\boldsymbol{x}, t_{n}\right)$.

2.3. Polyconvex energies for isotropic materials. If the free-energy function is frame invariant and isotropic relative to $\kappa$ then it is expressible as a function of the 
list $\left\{i_{1}, i_{2}, i_{3}, c\right\}$, where

$$
i_{1}=\operatorname{tr} \boldsymbol{U}, \quad i_{2}=\operatorname{tr} \boldsymbol{U}^{*}, \quad i_{3}=J_{F}
$$

are the principal invariants of the right-stretch tensor $\boldsymbol{U} \in \mathrm{Sym}^{+}$in the polar decomposition

$$
\boldsymbol{F}=\boldsymbol{R} \boldsymbol{U}
$$

of the deformation gradient, with $\boldsymbol{R} \in \mathrm{Orth}^{+}$. These are related to the more frequently used invariants

$$
I_{1}=\operatorname{tr} C, \quad I_{2}=\operatorname{tr} C^{*}, \quad I_{3}=J_{C}
$$

of the Cauchy-Green deformation tensor $\boldsymbol{C}=\boldsymbol{F}^{t} \boldsymbol{F}$ by the invertible transformation [Steigmann 2002]

$$
I_{1}=i_{1}^{2}-2 i_{2}, \quad I_{2}=i_{2}^{2}-2 i_{1} i_{3}, \quad I_{3}=i_{3}^{2} .
$$

However, grounds for using the $i_{k}$ in the formulation of constitutive equations, rather than $I_{k}$, are given below.

Because of the swelling constraint, any constitutive dependence of the energy on $i_{3}$ is induced by its dependence on $c$, provided that $c$ is strictly positive. The invariant $i_{3}$ is therefore redundant whenever the swelling constraint is operative, and the free-energy function is then expressible in the form

$$
\Psi(\boldsymbol{F}, c)=\psi\left(i_{1}, i_{2}, c\right) .
$$

Technically this function is defined on the manifold $\mathcal{M}$ in $\mathbb{R}^{10}$ defined by the swelling constraint $J_{F}-c=1$. However, the same function is well defined for states $(\boldsymbol{F}, c) \in \mathbb{R}^{10}$ that do not satisfy the constraint. Thus (31) may also serve as an extension of the free-energy function into $\mathbb{R}^{10}$, for purposes of differentiation in (19). The associated Piola stress is given by [Steigmann 2002]

$$
\boldsymbol{P}=\boldsymbol{R} \boldsymbol{\sigma},
$$

where

$$
\boldsymbol{\sigma}=\left(\frac{\partial \psi}{\partial i_{1}}+i_{1} \frac{\partial \psi}{\partial i_{2}}\right) \boldsymbol{I}-\frac{\partial \psi}{\partial i_{2}} \boldsymbol{U}-q \boldsymbol{U}^{*}
$$

is the (symmetric) Biot stress, and the chemical potential is

$$
\mu=\frac{\partial \psi}{\partial c}+q
$$

We remark that any alternative extension must, of course, reduce to (31) on the constraint manifold. Accordingly, because any trajectory $(\boldsymbol{F}(t), c(t)) \in \mathcal{M}$ has a derivative with respect to $t$ that lies in the tangent space to $M$ at the considered instant, it follows that the part of the derivative $\left(\Psi_{F}, \Psi_{c}\right)$ of the extended function, 
in the direction $\left(\boldsymbol{F}^{*},-1\right)$ orthogonal to $\mathcal{M}$, cannot be determined by inequality (18). Therefore, the use of an alternative extension affects only the as-yet-undetermined scalar field $q$. The extension (31) may therefore be used without loss of generality.

We may also use the swelling constraint to eliminate the concentration in favor of $i_{3}$ and thus express the energy as a function of $\left\{i_{1}, i_{2}, i_{3}\right\}$, in the manner of a conventional compressible solid [Treloar 1975; Pence and Tsai 2005; Pence and Tsai 2006; Deng and Pence 2010], albeit subject to the requirement $i_{3}>1$. In this interpretation we write (31) in the form

$$
\Psi^{\prime}(\boldsymbol{F})=\varphi\left(i_{1}, i_{2}, i_{3}\right),
$$

where $\Psi^{\prime}(\boldsymbol{F})=\Psi\left(\boldsymbol{F}, J_{F}-1\right)$ and, of course,

$$
\varphi\left(i_{1}, i_{2}, i_{3}\right)=\psi\left(i_{1}, i_{2}, i_{3}-1\right) .
$$

The minimum-energy argument of Section 2.2 applies to this surrogate compressible material.

In view of this it is natural to investigate constitutive functions that satisfy the well-known polyconvexity condition of Ball's existence theory for energy minimizers [1977]. Polyconvexity of $\Psi^{\prime}$ is the requirement that there exists a function

$$
\Phi\left(\boldsymbol{F}, \boldsymbol{F}^{*}, J_{F}\right)=\Psi^{\prime}(\boldsymbol{F}),
$$

in general non-unique [Podio-Guidugli 1991; Podio-Guidugli and Vergara-Caffarelli 1991], which is jointly convex in its arguments; we make the obvious choice

$$
\Phi\left(\boldsymbol{F}, \boldsymbol{F}^{*}, J_{F}\right)=\varphi\left\{\operatorname{tr}\left(\sqrt{\boldsymbol{F}^{t} \boldsymbol{F}}\right), \operatorname{tr}\left(\sqrt{\left(\boldsymbol{F}^{*}\right)^{t} \boldsymbol{F}^{*}}\right), J_{F}\right\} .
$$

If $\Phi$ is differentiable, then [Ball 1977]

$$
\begin{aligned}
\Psi^{\prime}(\overline{\boldsymbol{F}})-\Psi^{\prime}(\boldsymbol{F}) \geq \Phi_{\boldsymbol{F}}\left(\boldsymbol{F}, \boldsymbol{F}^{*}, J_{F}\right) \cdot(\overline{\boldsymbol{F}}-\boldsymbol{F}) & +\Phi_{\boldsymbol{F}^{*}}\left(\boldsymbol{F}, \boldsymbol{F}^{*}, J_{F}\right) \cdot\left(\overline{\boldsymbol{F}}^{*}-\boldsymbol{F}^{*}\right) \\
& +\Phi_{J_{F}}\left(\boldsymbol{F}, \boldsymbol{F}^{*}, J_{F}\right)\left(J_{\bar{F}}-J_{F}\right)
\end{aligned}
$$

for all deformation gradients $\boldsymbol{F}$ and $\overline{\boldsymbol{F}}$. Here we use

$$
\left(i_{1}\right)_{\boldsymbol{F}}=\left(i_{2}\right)_{F^{*}}=\boldsymbol{R}
$$

[Steigmann 2003] to conclude that

$$
\Phi_{\boldsymbol{F}}=\frac{\partial \varphi}{\partial i_{1}} \boldsymbol{R} \quad \text { and } \quad \Phi_{\boldsymbol{F}^{*}}=\frac{\partial \varphi}{\partial i_{2}} \boldsymbol{R} .
$$

Polyconvexity ensures that the pointwise values of the free energy fulfill the (non-local) quasiconvexity condition, which is always satisfied by energy minimizers [Ball 1977]. Further, it guarantees the sequential weak lower semi-continuity of the potential energy functional which, together with coercivity of the free-energy function, ensures the existence of energy-minimizing deformations belonging to 
an appropriate Sobolev space. Here, coercivity refers to the restriction [Ball 1977; Steigmann 2003]

$$
\varphi\left(i_{1}, i_{2}, i_{3}\right) \geq k_{1}\left(i_{1}^{p}+i_{2}^{q}+i_{3}^{r}\right)+k_{2},
$$

where $p \geq 2, q \geq p /(p-1), r>1$ and $k_{1}, k_{2}$ are constants with $k_{1}>0$. Moreover, polyconvexity is free from the well-known objections [Ball 1977] raised against ordinary convexity of the free energy with respect to the deformation gradient, a condition which also implies quasiconvexity. However, Ball's existence theory is not immediately applicable here because of inequality (17); whereas that theory relies on a hypothesis about the behavior of the free-energy function in the limit $J_{F} \rightarrow 0$.

Thus our restriction to polyconvex energies is motivated by the fact that quasiconvexity, which is necessary for energy minimizers, is thereby assured. Further, the coercivity condition, while an integral part of Ball's theory, is evidently restrictive as a number of explicit solutions to equilibrium boundary-value problems have been obtained using non-coercive free energies [Carroll 1988]. Therefore we do not impose coercivity.

By [Steigmann 2003], the function $\Psi^{\prime}(\boldsymbol{F})$ defined by (37) and (38) is polyconvex if and only if

$\varphi$ is a convex function of all three arguments jointly, and $\varphi$ is a nondecreasing function of $i_{1}$ and $i_{2}$;

that is, if and only if

$$
\varphi\left(\bar{l}_{1}, \bar{l}_{2}, \bar{l}_{3}\right)-\varphi\left(i_{1}, i_{2}, i_{3}\right) \geq\left(\bar{l}_{1}-i_{1}\right) \frac{\partial \varphi}{\partial i_{1}}+\left(\bar{l}_{2}-i_{2}\right) \frac{\partial \varphi}{\partial i_{2}}+\left(\bar{l}_{3}-i_{3}\right) \frac{\partial \varphi}{\partial i_{3}}
$$

together with

$$
\frac{\partial \varphi}{\partial i_{1}} \geq 0 \quad \text { and } \quad \frac{\partial \varphi}{\partial i_{2}} \geq 0
$$

in which $i_{k}$ and $\bar{l}_{k}$, respectively, are the invariants associated with $\boldsymbol{F}$ and $\overline{\boldsymbol{F}}$ and the derivatives are evaluated at $i_{k}$. Sufficiency follows from (41) and the fact that $i_{1}$ and $i_{2}$ are convex functions of $\boldsymbol{F}$ and $\boldsymbol{F}^{*}$, respectively [Steigmann 2003], i.e.,

$$
i_{1}(\overline{\boldsymbol{F}})-i_{1}(\boldsymbol{F}) \geq \boldsymbol{R}(\boldsymbol{F}) \cdot(\overline{\boldsymbol{F}}-\boldsymbol{F}) \quad \text { and } \quad i_{2}\left(\overline{\boldsymbol{F}}^{*}\right)-i_{2}\left(\boldsymbol{F}^{*}\right) \geq \boldsymbol{R}\left(\boldsymbol{F}^{*}\right) \cdot\left(\overline{\boldsymbol{F}}^{*}-\boldsymbol{F}^{*}\right) .
$$

A proof of the necessity of (44) and (45) for polyconvexity is given here in the Appendix. In this regard it is important to note that (43) is equivalent to polyconvexity in respect of the function defined by (38), but may not be if alternative choices are adopted. This is due to the fact that the function $\Phi$ defined by (37) is not unique.

The simplicity of the polyconvexity criteria (43) supports our preference for a constitutive formulation based on the stretch tensor. 
Of particular relevance to the present work is the free-energy function

$$
\Psi_{(c)}(\boldsymbol{F} ; \boldsymbol{x})=\Psi(\boldsymbol{F}, c(\boldsymbol{x})),
$$

obtained by fixing the concentration field at $c(\boldsymbol{x})$, say, which may be specified as an arbitrary function taking non-negative values. We expect that Ball's theory for incompressible materials, adapted to accommodate the assignment of $J_{F}(\boldsymbol{x})$ in accordance with the swelling constraint, may yield the existence of minimizers in this case.

Remark. This free-energy function pertains to a gel in which diffusion has ceased and the volumetric deformation $J_{F}(\boldsymbol{x})$ is fixed. Equation (2), with (19) $)_{2},(22)$ and $\dot{c}=0$, then becomes a restriction on the function $q(\boldsymbol{x})$. The associated flux $\boldsymbol{m}$ is divergence-free and the net (integrated) flux through the boundary $\partial \kappa$ vanishes. The argument in Section 2.2 about minimum-energy states is applicable under the slightly stronger condition that $\boldsymbol{m} \cdot \boldsymbol{n}$ and $\mu$ vanish pointwise on complementary parts of the boundary; these yield data for the determination of $q(\boldsymbol{x})$, if desired.

The function $\Psi_{(c)}$ is polyconvex at $x^{*} \in \kappa$ if and only if there exists a convex function

$$
\Phi_{\left(c^{*}\right)}\left(\boldsymbol{F}, \boldsymbol{F}^{*}\right)=\Psi\left(\boldsymbol{F}, c^{*}\right),
$$

where $c^{*}=c\left(\boldsymbol{x}^{*}\right)$; that is, if and only if

$$
\begin{aligned}
\Psi_{(c)}\left(\overline{\boldsymbol{F}} ; \boldsymbol{x}^{*}\right)- & \Psi_{(c)}\left(\boldsymbol{F} ; \boldsymbol{x}^{*}\right) \\
& \geq \Phi_{\left(c^{*}\right) \boldsymbol{F}}\left(\boldsymbol{F}, \boldsymbol{F}^{*}\right) \cdot(\overline{\boldsymbol{F}}-\boldsymbol{F})+\Phi_{\left(c^{*}\right) \boldsymbol{F}^{*}}\left(\boldsymbol{F}, \boldsymbol{F}^{*}\right) \cdot\left(\overline{\boldsymbol{F}}^{*}-\boldsymbol{F}^{*}\right) .
\end{aligned}
$$

In the present circumstances we take this function to be

$$
\Phi_{(c)}\left(\boldsymbol{F}, \boldsymbol{F}^{*}\right)=\psi\left\{\operatorname{tr}\left(\sqrt{\boldsymbol{F}^{t} \boldsymbol{F}}\right), \operatorname{tr}\left(\sqrt{\left(\boldsymbol{F}^{*}\right)^{t} \boldsymbol{F}^{*}}\right), c\right\} .
$$

The inequality does not involve the determinant of the deformation gradient; this is fixed at the value $1+c^{*}$ by the swelling constraint. Necessary and sufficient conditions in this case are [Steigmann 2003]:

$\psi$ is a convex function of $i_{1}$ and $i_{2}$ jointly, and

$\psi$ is a nondecreasing function of $i_{1}$ and $i_{2}$;

that is,

$$
\psi\left(\bar{l}_{1}, \bar{l}_{2}, c^{*}\right)-\psi\left(i_{1}, i_{2}, c^{*}\right) \geq\left(\bar{l}_{1}-i_{1}\right) \frac{\partial \psi}{\partial i_{1}}+\left(\bar{l}_{2}-i_{2}\right) \frac{\partial \psi}{\partial i_{2}},
$$

together with

$$
\frac{\partial \psi}{\partial i_{1}} \geq 0 \quad \text { and } \quad \frac{\partial \psi}{\partial i_{2}} \geq 0
$$


in which $i_{k}$ and $\bar{l}_{k}$, respectively, are the invariants associated with $\boldsymbol{F}$ and $\overline{\boldsymbol{F}}$ and the derivatives are again evaluated at $i_{k}$. Sufficiency is proved in [Steigmann 2003] whereas the proof of necessity given in the Appendix applies here as well.

2.4. Local free swelling. Consider a subvolume $\pi \subset \kappa$ with $\boldsymbol{p}$ and $\mu \boldsymbol{m} \cdot \boldsymbol{n}$ vanishing everywhere on $\partial \pi$. The foregoing argument about the decay of energy is thus applicable to $\pi$ with the load potential equal to a constant. This is the free-swelling problem, and plays a central role in [Treloar 1975; Pence and Tsai 2005; Hong et al. 2008; Duda et al. 2010; Chester and Anand 2011], where it is used to specify constitutive information; i.e., restrictions on the response of the gel at points in $\kappa$. To make contact with these ideas, we make repeated use of the following simple result: If $F(\boldsymbol{x})$ is continuous and $M V(F)$ is the mean value of $F$ in $\pi$, then there is a point $\boldsymbol{x}^{*} \in \pi$ such that $F\left(\boldsymbol{x}^{*}\right)=M V(F)$. Further, if $d(\pi) \rightarrow 0$, where $d(\pi)=$ $\sup _{x_{1}, x_{2} \in \pi}\left|x_{1}-x_{2}\right|$ is the diameter of $\pi$, then $\left|x-x^{*}\right| \rightarrow 0$ for all $x \in \pi$ and the continuity of $F$ implies that $F(\boldsymbol{x}) \rightarrow F\left(\boldsymbol{x}^{*}\right)$. For example, the well-known mean-stress theorem, following from (4) and (6), yields

$$
M V(\boldsymbol{P})=\int_{\partial \pi} \boldsymbol{p} \otimes \boldsymbol{x} d a,
$$

which vanishes in the free-swelling problem. Accordingly, $\boldsymbol{P}(\boldsymbol{x}) \rightarrow \mathbf{0}$ as $d(\pi) \rightarrow 0$, implying that the pointwise values of the stress field may be brought arbitrarily close to zero by making the diameter of $\pi$ correspondingly small against any available length scale. In the same way, by integrating $\mu \boldsymbol{m} \cdot \boldsymbol{n}(=0)$ over $\partial \pi$, we find, using the divergence theorem and the diffusive balance, that

$$
M V(\mu \dot{c})=M V(\boldsymbol{m} \cdot D \mu) \leq 0 .
$$

Therefore, if $d(\pi) \rightarrow 0$ it follows that

$$
\mu \dot{c} \leq 0,
$$

pointwise in $\pi$.

In the present setting, the condition on stress reduces to $\sigma=\mathbf{0}$; i.e.,

$$
\left(\frac{\partial \psi}{\partial i_{1}}+i_{1} \frac{\partial \psi}{\partial i_{2}}\right) \boldsymbol{I}-\frac{\partial \psi}{\partial i_{2}} \boldsymbol{U}=q \boldsymbol{U}^{*} .
$$

The trace yields

$$
q i_{2}=3 \frac{\partial \psi}{\partial i_{1}}+2 i_{1} \frac{\partial \psi}{\partial i_{2}}
$$

which furnishes $q$ in terms of $i_{1}, i_{2}$ and $c$. If this state is at least asymptotically stable then it minimizes the energy; i.e.,

$$
M V[\Psi(\overline{\boldsymbol{F}}, \bar{c})-\Psi(\boldsymbol{F}, c)] \geq 0,
$$


where $\overline{\boldsymbol{F}}(\boldsymbol{x})$ and $\bar{c}(\boldsymbol{x})$ are any deformation and concentration fields consistent with the balance laws and boundary conditions. Accordingly, if $d(\pi) \rightarrow 0$ then

$$
\Psi(\overline{\boldsymbol{F}}, \bar{c}) \geq \Psi(\boldsymbol{F}, c)
$$

again pointwise.

Two definitions of the free-swelling problem are evident:

(a) In the first, which is tacitly adopted in [Pence and Tsai 2005; Chester and Anand 2011], the flux $\boldsymbol{m} \cdot \boldsymbol{n}$ is unrestricted on $\partial \pi$; this requires $\mu$ to vanish there, and the local inequality (56) is satisfied if $d(\pi) \rightarrow 0$, with $\dot{c}$ unrestricted. This in turn requires that $\mu=0$ pointwise, ensuring that the condition on the boundary is satisfied. Equation (34) gives $q=-\partial \psi / \partial c$, while (58) reduces to

$$
3 \frac{\partial \varphi}{\partial i_{1}}+2 \frac{\partial \varphi}{\partial i_{2}}+i_{2} \frac{\partial \varphi}{\partial i_{3}}=0
$$

where we have used the connection (36) pertaining to the equivalent compressible material, in which the stress is given by (33) with $\psi$ replaced by $\varphi$ and $c$ by $i_{3}-1$. This is effectively the free-swelling condition given in Equation (7) of [Deng and Pence 2010], yielding an equation for $c$. We note that our polyconvexity criteria $(43)_{2}$ then require that $\partial \varphi / \partial i_{3} \leq 0$, which is compatible with $(43)_{1}$.

(b) In the second definition of free swelling, proposed here, the diffusive flux $\boldsymbol{m} \cdot \boldsymbol{n}$ vanishes pointwise on $\partial \pi$ and the argument leading to (55), with $\mu \boldsymbol{m}$ replaced by $\boldsymbol{m}$, yields

$$
\frac{d}{d t} M V(c)=0, \quad \text { so that } \quad M V(c)=c^{*},
$$

a constant. Accordingly, if $d(\pi) \rightarrow 0$ then

$$
c=c^{*},
$$

pointwise. This implies $\dot{c}=0$, ensuring that (56) is satisfied. Inequality (60) reduces to

$$
\Psi_{\left(c^{*}\right)}(\overline{\boldsymbol{F}}) \geq \Psi_{\left(c^{*}\right)}(\boldsymbol{F}) ; \quad J_{\bar{F}}=J_{F}=1+c^{*},
$$

where $\Psi_{(c)}$ is defined by (47).

In the literature on isotropic elastomers [Treloar 1975; Pence and Tsai 2005; Hong et al. 2008; Duda et al. 2010; Chester and Anand 2011] we find the universal assumption that the free-swelling deformation is a pure equi-triaxial stretch; i.e., that

$$
\boldsymbol{F}=\lambda \boldsymbol{I}
$$


for some $\lambda>0$, yielding $\boldsymbol{U}=\boldsymbol{F}$. Then, Equation (58) reduces to

$$
\frac{\partial \psi}{\partial i_{1}}+2 \lambda \frac{\partial \psi}{\partial i_{2}}=\lambda^{2} q
$$

in which the derivatives are evaluated at

$$
i_{1}=3 \lambda \quad \text { and } \quad i_{2}=3 \lambda^{2} .
$$

The swelling constraint yields

$$
\lambda^{3}=1+c^{*}
$$

and (66) and (34) yield unique values of $q$ and $\mu$. We have thus established the existence of a state that satisfies the local free-swelling problem in which concentration is assigned.

If the solution described by (65)-(68) is to be stable, it must satisfy (64). To investigate this we follow [Ogden 1984, p. 110] and decompose the deformation $\overline{\boldsymbol{F}}$ of the comparison state in the form

$$
\overline{\boldsymbol{F}}=\overline{\boldsymbol{R}} \overline{\boldsymbol{U}},
$$

in which $\overline{\boldsymbol{R}} \in \mathrm{Orth}^{+}$and

$$
\begin{aligned}
\overline{\boldsymbol{U}} & =\sum \bar{\lambda}_{i} \boldsymbol{u}_{i} \otimes \boldsymbol{u}_{i} \\
& =(\bar{\lambda} \boldsymbol{I})\left(s \boldsymbol{u}_{1} \otimes \boldsymbol{u}_{1}+s^{-1} \boldsymbol{u}_{2} \otimes \boldsymbol{u}_{2}+\boldsymbol{u}_{3} \otimes \boldsymbol{u}_{3}\right)\left[t^{-1 / 2}\left(\boldsymbol{u}_{1} \otimes \boldsymbol{u}_{1}+\boldsymbol{u}_{2} \otimes \boldsymbol{u}_{2}\right)+t \boldsymbol{u}_{3} \otimes \boldsymbol{u}_{3}\right],
\end{aligned}
$$

where $\bar{\lambda}_{i}(>0)$ are the principal stretches, $\left\{\boldsymbol{u}_{i}\right\}$ are the orthonormal principal axes of $\overline{\boldsymbol{U}}$ and the factors correspond to a pure equi-triaxial stretch of amount $\bar{\lambda}(>0)$, a pure shear of amount $s(>0)$ and an isochoric uniaxial extension of amount $t(>0)$ with accompanying lateral contraction. These are coaxial and so may be composed in any order. The principal stretches are

$$
\bar{\lambda}_{1}=\bar{\lambda} s t^{-1 / 2}, \quad \bar{\lambda}_{2}=\bar{\lambda} s^{-1} t^{-1 / 2}, \quad \bar{\lambda}_{3}=\bar{\lambda} t,
$$

which may be inverted to yield

$$
\bar{\lambda}=\left(\bar{\lambda}_{1} \bar{\lambda}_{2} \bar{\lambda}_{3}\right)^{1 / 3}, \quad t=\bar{\lambda}_{3}^{2 / 3}\left(\bar{\lambda}_{1} \bar{\lambda}_{2}\right)^{-1 / 3}, \quad s=\left(\bar{\lambda}_{1} / \bar{\lambda}_{2}\right)^{1 / 2} .
$$

Accordingly, (71) affords a general representation of any state of stretch and the decomposition (70) entails no loss of generality [Ogden 1984]. The corresponding invariants are

$$
\bar{\imath}_{1}=\bar{\lambda}\left[\left(s+s^{-1}\right) t^{-1 / 2}+t\right], \quad \bar{\imath}_{2}=\bar{\lambda}^{2}\left[\left(s+s^{-1}\right) t^{1 / 2}+t^{-1}\right], \quad \bar{\imath}_{3}=\bar{\lambda}^{3} .
$$


It is instructive to insert these expressions into the polyconvexity criteria. Because $c$ is fixed at the value $c^{*}$ in inequality (64), the relevant polyconvexity condition is (49), in which $\bar{\lambda}=\lambda$. Accordingly, (52) yields

$$
\Psi_{\left(c^{*}\right)}(\overline{\boldsymbol{F}})-\Psi_{\left(c^{*}\right)}(\boldsymbol{F}) \geq \lambda f(s, t) \frac{\partial \psi}{\partial i_{1}}+\lambda^{2} f(s, u) \frac{\partial \psi}{\partial i_{2}},
$$

where $u=t^{-1}$, and, for $x$ and $y$ positive,

$$
f(x, y)=\left(x+x^{-1}\right) y^{-1 / 2}+y-3 .
$$

The derivatives in (74) are evaluated at the invariants given in (67), with (68).

The function $f$ is stationary at $(x, y)=(1,1)$. Its Hessian matrix there is easily shown to be positive definite, so that $f$ has a local minimum, equal to zero, at $(1,1)$. At this point both the pure shear and uniaxial extension of (70) reduce to the identity, and $\overline{\boldsymbol{U}}=\boldsymbol{U}(=\lambda \boldsymbol{I})$. It then follows from (74) that $\Psi_{\left(c^{*}\right)}$ has a local minimum at the equi-triaxial stretch defined by (67) and (68). In fact the minimum is global, as $f \geq 0$ for all values of its arguments. This claim is easily proved by observing that it is equivalent to the inequality $x+x^{-1} \geq(3-y) y^{1 / 2}$, the truth of which follows from the fact that the left-hand side has a strict global minimum, equal to 2, at $x=1$; whereas the right-hand side has a strict global maximum, also equal to 2 , at $y=1$. Thus, $f(1,1)=0$ and $f(x, y)>0$ for all $(x, y) \neq(1,1)$ with $x$ and $y$ positive. In particular, inequality (74) and the strict versions of (53) then imply that (64) holds in the strict sense for all $\overline{\boldsymbol{U}} \neq \boldsymbol{U}$ such that $J_{\bar{F}}=J_{F}$.

We have shown that the polyconvexity criteria (51) imply that a state defined by (57) and (65) furnishes an optimal solution to the free swelling problem associated with a given concentration. This result is perhaps surprising. For, although polyconvexity implies quasiconvexity, the latter is not known to imply (64). In fact the optimality of the solution (65) follows from the weaker restriction of rank-one convexity. This is demonstrated in the Appendix.

Our result justifies the local decomposition [Chester and Anand 2011]

$$
\boldsymbol{F}=\boldsymbol{H} \boldsymbol{G},
$$

in which

$$
\boldsymbol{G}=(1+c)^{1 / 3} \boldsymbol{I} \quad \text { with } c \geq 0,
$$

is the free-swelling deformation associated with $c$, yielding a swollen stress-free local configuration $\kappa_{c}$ followed by an elastic deformation $\boldsymbol{H}$ that gives rise to stress. Indeed, if such $\boldsymbol{G}$ were not energetically optimal, then theories based on (76) might well yield predictions that are sub-optimal and perhaps even unstable. We observe that (76) and (77) are consistent with the swelling constraint (16) if and only if

$$
J_{H}=1 \text {. }
$$


Using the foregoing procedure, the free-swelling state can be achieved at all points of the gel provided that $\kappa$ is divided into an arbitrarily large number of subvolumes, each of which satisfies the free-swelling problem. In general, the local configurations resulting from this operation cannot be made congruent in three-dimensional space in the absence of strain; that is, they do not necessarily fit together to form a connected whole in Euclidean space. Instead, the union of such states is to be regarded as a smooth three-dimensional manifold, whose tangent space at a given value of $c$ is identified with $\kappa_{c}$. The fact that this manifold is generally non-Euclidean implies that $\boldsymbol{G}$ is not the gradient of any position field. Accordingly, it does not satisfy the compatibility condition which follows from the existence of such a field. Of course, $\boldsymbol{F}$, being the gradient of the map $\boldsymbol{\chi}$, is necessarily compatible. The incompatibility of $\boldsymbol{G}$ then implies that $\boldsymbol{H}$ is likewise incompatible. That such a formalism is necessary follows from the fact that $\boldsymbol{G}$ is compatible if and only if the field $c(x, t)$ is uniform. In this case the equilibrium equation, diffusive balance and boundary/initial conditions yield an overdetermined problem for the fields $\chi(\boldsymbol{x}, t)$ and $q(\boldsymbol{x}, t)$, having no solution except in special circumstances.

2.5. Using $\kappa_{c}$ as reference. The decomposition (76) suggests the use of $\kappa_{c}$ as reference when formulating constitutive equations [Chester and Anand 2011]. For example, given a deformation field $\chi(\boldsymbol{x}, t)$ the free-energy function per unit volume of $\kappa_{c}$ is $W(\boldsymbol{F} \boldsymbol{K}, c)=J_{K} \Psi(\boldsymbol{F}, c)$, where $\boldsymbol{K}=\boldsymbol{G}^{-1}$. Accordingly,

$$
\Psi(\boldsymbol{F}, c)=(1+c) W(\boldsymbol{H}, c), \quad \text { where } \quad \boldsymbol{H}=(1+c)^{-1 / 3} \boldsymbol{F} .
$$

This decomposition shows that the present model is subsumed, at fixed $c$, under Noll's theory of materially uniform bodies [Noll 1967], which has dramatically advanced the development of theories of plasticity and continuously distributed defects. Here, because the local change of reference from $\kappa$ to $\kappa_{c}$ entails a pure dilation (cf. (77)), it does not affect the symmetry group of the gel. This is a simple consequence of Noll's rule [Truesdell 1977] connecting the symmetry groups associated with local references. Accordingly, the material is isotropic with respect to both $\kappa$ and $\kappa_{c}$. This fact, the constraint (16) and the invariance of $W$ under superposed rotations - a property inherited from $\Psi$-imply that the strain energy $W$ depends on $\boldsymbol{H}$ via the list $\left\{h_{1}, h_{2}\right\}$, where

$$
h_{1}=\operatorname{tr} \boldsymbol{U}_{H} \quad \text { and } \quad h_{2}=\operatorname{tr} \boldsymbol{U}_{H}^{*} .
$$

Here $\boldsymbol{U}_{H}$ is the symmetric, positive right-stretch tensor in the polar factorization of $\boldsymbol{H}$. From $(79)_{2}$, the rotation in this factorization is simply $\boldsymbol{R}$, the rotation associated with $\boldsymbol{F}$. It follows that $\boldsymbol{U}_{H}=(1+c)^{-1 / 3} \boldsymbol{U}$; therefore,

$$
h_{1}=(1+c)^{-1 / 3} i_{1} \text { and } h_{2}=(1+c)^{-2 / 3} i_{2} .
$$


These in turn yield $W(\boldsymbol{H}, c)=w\left(h_{1}, h_{2}, c\right)$ for some function $w$, which may be used to write (79) in the form

$$
\psi\left(i_{1}, i_{2}, c\right)=(1+c) w\left(h_{1}, h_{2}, c\right) .
$$

Using this with (81), it is straightforward to show that the polyconvexity criteria (51) are satisfied if and only if

$w$ is a convex function of $h_{1}$ and $h_{2}$ jointly, and

$w$ is a nondecreasing function of $h_{1}$ and $h_{2}$,

and this in turn implies that the function $W_{(c)}(\boldsymbol{H})=W(\boldsymbol{H}, c)$, associated with fixed $c$, is polyconvex [Steigmann 2003]. Thus, there is a function $G_{(c)}\left(\boldsymbol{H}, \boldsymbol{H}^{*}\right)=$ $W_{(c)}(\boldsymbol{H})$ such that

$$
\begin{array}{r}
W_{(c)}(\overline{\boldsymbol{H}})-W_{(c)}(\boldsymbol{H}) \\
\quad \geq G_{(c) \boldsymbol{H}}\left(\boldsymbol{H}, \boldsymbol{H}^{*}\right) \cdot(\overline{\boldsymbol{H}}-\boldsymbol{H})+G_{(c) \boldsymbol{H}^{*}}\left(\boldsymbol{H}, \boldsymbol{H}^{*}\right) \cdot\left(\overline{\boldsymbol{H}}^{*}-\boldsymbol{H}^{*}\right) .
\end{array}
$$

In the present circumstances we take $G_{(c)}\left(\boldsymbol{H}, \boldsymbol{H}^{*}\right)=w\left(h_{1}, h_{2}, c\right)$ (cf. (50)), yielding $\boldsymbol{G}_{(c) \boldsymbol{H}}=\left(\partial w / \partial h_{1}\right) \boldsymbol{R}$ and $\boldsymbol{G}_{(c) \boldsymbol{H}^{*}}=\left(\partial w / \partial h_{2}\right) \boldsymbol{R}$. Indeed, using (81), (82) and (41) it is straightforward to show that (84) is equivalent to inequality (49). This furnishes an explicit example of the general fact that polyconvexity is preserved under any fixed change of reference [Neff 2003, Lemma 6.5, p. 260]. Using the results of the previous subsection, we conclude that any distortion; i.e., any $\boldsymbol{U}_{H} \neq \boldsymbol{I}$ with $J_{H}=1$, entails an energetic cost if $w$ satisfies (83).

2.6. Constitutive specification of the diffusive flux. A well-known representation theorem for isotropic functions [Noll 1970] furnishes the canonical form of the mobility tensor for materials exhibiting holohedral (as distinct from hemihedral) isotropy. Here, we combine this theorem with the Cayley-Hamilton formula to conclude that

$$
\boldsymbol{M}=\alpha_{0} \boldsymbol{I}+\alpha_{1} \boldsymbol{U}+\alpha_{2} \boldsymbol{U}^{2},
$$

where $\alpha_{0,1,2}$ are functions of $i_{1,2}$ and $c$, arranged to ensure that the mobility is negative definite. The swelling constraint implies that $i_{3}$ is redundant. This representation furnishes the referential diffusive flux $\boldsymbol{m}$ defined by (22).

In [Chester and Anand 2011] the diffusive flux is expressed in terms of variables pertaining to the local stress-free swollen configuration $\kappa_{c}$. The operative diffusive flux is the push-forward

$$
\boldsymbol{m}_{c}=J_{G}^{-1} \boldsymbol{G m}
$$

of the referential flux $\boldsymbol{m}$. Using (22) and (77), this is easily shown to yield

$$
\boldsymbol{m}_{c}=\left(\gamma_{0} \boldsymbol{I}+\gamma_{1} \boldsymbol{U}_{H}+\gamma_{2} \boldsymbol{U}_{H}^{2}\right) \boldsymbol{H}^{t}(\operatorname{grad} \mu)
$$


where grad is the spatial gradient based on position $\boldsymbol{y}$ and $\gamma_{0,1,2}$ are functions of the invariants $h_{1,2}$ and $c$. This is an isotropic function jointly of $\boldsymbol{U}_{H}$ and the pushforward to $\kappa_{c}$ of the referential chemical-potential gradient, namely, $\boldsymbol{H}^{t}(\operatorname{grad} \mu)=$ $\boldsymbol{G}^{-t} D \mu$.

Chester and Anand [2011] state that isotropy of the constitutive equation for a single diffusive flux vector should be imposed relative to both $\kappa$ and $\kappa_{c}$. This restriction implies that the mobility tensor is purely spherical; i.e., that it is proportional to the identity, as they point out. However, we have shown that isotropy is preserved under the transformation $\kappa \rightarrow \kappa_{c}$, provided that the appropriate flux vector is used. Thus we conclude that the imposition in [Chester and Anand 2011] of the dual requirement on the constitutive equation for a single diffusive flux vector is not appropriate. Instead, we observe that the preservation of isotropy is due to the fact that the transformation $\kappa \rightarrow \kappa_{c}$ is a pure dilation. According to Noll's theorem [Truesdell 1977], these do not alter the symmetry group. When using this theorem it is essential to account for the induced change in the referential variables (cf. (86)).

\section{Two-dimensional theory}

The two-dimensional version of the foregoing theory, applicable to plane-strain deformations, is substantially simpler. Here we use the same notation as before with the stipulation that all tensors are regarded as linear maps from $E^{2}$ to itself. Thus the model discussed is inherently two dimensional. We present a synopsis of the main results in this case, emphasizing those features that differ from the corresponding three-dimensional theory.

The arguments leading to (19) and (32) remain unaltered, and thus yield

$$
\boldsymbol{P}=\boldsymbol{R} \boldsymbol{\sigma}, \quad \text { with } \boldsymbol{\sigma}=\frac{\partial \varphi}{\partial i} \boldsymbol{I}-q \boldsymbol{U}^{*},
$$

where

$$
i=\operatorname{tr} U
$$

is one of the two independent isotropic invariants. The other, $\operatorname{det} \boldsymbol{U}$, is fixed by the swelling constraint, which carries over without modification, except of course that all reference to volume is replaced here by area. The relation (34) connecting the chemical potential, free energy and Lagrange multiplier is also unaltered.

In two dimensions we have [Steigmann 2002]

$$
i \boldsymbol{R}=\boldsymbol{F}+\boldsymbol{F}^{*},
$$

and therefore

$$
\boldsymbol{P}=i^{-1}\left(\frac{\partial \psi}{\partial i}\right)\left(\boldsymbol{F}+\boldsymbol{F}^{*}\right)-q \boldsymbol{F}^{*},
$$


which is required to satisfy $\operatorname{div} \boldsymbol{P}=\mathbf{0}$ in $\kappa$ together with any traction data on $\partial \kappa$, where div is the two-dimensional divergence on $\kappa$.

The diffusive balance (2) takes the form

$$
\dot{c}+\operatorname{div}[\boldsymbol{M}(\boldsymbol{U}, c) \nabla \mu]=0,
$$

in which $\nabla$ is the two-dimensional gradient and, by the two-dimensional representation theorem for isotropic functions,

$$
\boldsymbol{M}(\boldsymbol{U}, c)=\beta_{0}(i, c) \boldsymbol{I}+\beta_{1}(i, c) \boldsymbol{U},
$$

in which $\boldsymbol{I}$ is the identity for 2-space and the functions $\beta_{0,1}$ are restricted by the requirement that $\boldsymbol{M}$ be negative definite. To obtain $\boldsymbol{U}$ from the deformation gradient we use the two-dimensional Cayley-Hamilton formula

$$
\boldsymbol{U}=i^{-1}[(1+c) \boldsymbol{I}+\boldsymbol{C}], \quad \text { where } \boldsymbol{C}=\boldsymbol{F}^{t} \boldsymbol{F},
$$

in which the swelling constraint has been imposed.

The operative polyconvexity condition, replacing (51), is

$$
\psi(i, c) \text { is a convex, nondecreasing function of } i \text {. }
$$

This is necessary and sufficient for $\Psi_{(c)}(\boldsymbol{F})=\psi(i(\boldsymbol{F}), c)$ to be polyconvex [Steigmann 2003].

The free-swelling problem, in which $c$ is assigned, is again solved by deformations of the form $\boldsymbol{F}=\lambda \boldsymbol{I}$, but now with

$$
\lambda^{2}=1+c
$$

Equation (66) is replaced by

$$
q=\lambda^{-1} \frac{\partial \psi}{\partial i},
$$

in which the derivative is evaluated at $i=2 \lambda$, and (34) then furnishes a unique value of $\mu$.

Polyconvexity again ensures the optimality of this solution to the free-swelling problem. To see this we use (95) to obtain

$$
\Psi_{(c)}(\overline{\boldsymbol{F}})-\Psi_{(c)}(\boldsymbol{F}) \geq(\bar{l}-i) \frac{\partial \psi}{\partial i},
$$

in which the derivative is evaluated at $i=2 \lambda$. The gradient $\overline{\boldsymbol{F}}$ may, without loss of generality, be decomposed in the form (69), where

$$
\overline{\boldsymbol{U}}=(\bar{\lambda} \boldsymbol{I})\left(s \boldsymbol{u}_{1} \otimes \boldsymbol{u}_{1}+s^{-1} \boldsymbol{u}_{2} \otimes \boldsymbol{u}_{2}\right)
$$


is the composition of an areal dilation of amount $\bar{\lambda}$ and a pure shear of amount $s(>0)$. The associated principal stretches are $\bar{\lambda}_{1}=\bar{\lambda} s$ and $\bar{\lambda}_{2}=\bar{\lambda} s^{-1}$, yielding

$$
\bar{\lambda}=\bar{\lambda}_{1} \bar{\lambda}_{2} \quad \text { and } \quad s=\left(\bar{\lambda}_{1} / \bar{\lambda}_{2}\right)^{1 / 2} .
$$

Here we impose $\bar{\lambda}=\lambda$ in accordance with the swelling constraint, obtaining $\bar{\imath}=$ $\lambda\left(s+s^{-1}\right)$. Inequality (98) reduces to

$$
\Psi_{(c)}(\overline{\boldsymbol{F}})-\Psi_{(c)}(\boldsymbol{F}) \geq \lambda\left(s+s^{-1}-2\right) \frac{\partial \psi}{\partial i},
$$

which is non-negative by virtue of (95) and the fact that $s+s^{-1}$ has an isolated minimum, equal to 2 , at $s=1$, corresponding to $\overline{\boldsymbol{U}}=\boldsymbol{U}$.

This result justifies the decomposition (cf. (76))

$$
\boldsymbol{F}=\boldsymbol{H} \boldsymbol{G},
$$

in which

$$
\boldsymbol{G}=(1+c)^{1 / 2} \boldsymbol{I} \quad \text { with } c \geq 0,
$$

and

$$
J_{H}=1,
$$

by virtue of the swelling constraint.

The free energy, per unit reference area, is then expressible in the form

$$
\psi(i, c)=(1+c) w(h, c), \quad \text { where } h=i / \sqrt{1+c},
$$

is the trace of $\boldsymbol{U}_{H}$, the stretch factor in the polar decomposition of $\boldsymbol{H}$, and $w$ is the free energy per unit area of the swollen elastomer. It is easy to show that the polyconvexity criterion (95) holds if and only if the same restriction applies to $w$; that is, $w(h, c)$ is a convex, nondecreasing function of $h$.

\section{Appendix}

In [Steigmann 2003] the conditions (43) are shown to be sufficient for polyconvexity. The proof of necessity is given here.

The necessity of (44) follows immediately from (39), (40) and (41) by selecting a deformation $\overline{\boldsymbol{F}}$ for which $\boldsymbol{R}(\overline{\boldsymbol{F}})=\boldsymbol{R}(\boldsymbol{F})$, and hence $\boldsymbol{R}\left(\overline{\boldsymbol{F}}^{*}\right)=\boldsymbol{R}\left(\boldsymbol{F}^{*}\right)$; these yield $\boldsymbol{R} \cdot(\overline{\boldsymbol{F}}-\boldsymbol{F})=\bar{\imath}_{1}-i_{1}$ and $\boldsymbol{R} \cdot\left(\overline{\boldsymbol{F}}^{*}-\boldsymbol{F}^{*}\right)=\bar{\imath}_{2}-i_{2}$, respectively, thereby reducing (39) to (44).

To demonstrate the necessity of (45) we use the fact that polyconvexity implies rank-one convexity [Ball 1977], which is equivalent to the inequality

$$
\Psi^{\prime}(\overline{\boldsymbol{F}})-\Psi^{\prime}(\boldsymbol{F}) \geq \boldsymbol{P}(\boldsymbol{F}) \cdot(\overline{\boldsymbol{F}}-\boldsymbol{F}), \quad \text { with } \overline{\boldsymbol{F}}-\boldsymbol{F}=\boldsymbol{a} \otimes \boldsymbol{b},
$$


for arbitrary $\boldsymbol{a}$ and $\boldsymbol{b}$. For isotropic materials, (32), (33), (34) and (36) may be used to express this in the form

$$
\Psi^{\prime}(\overline{\boldsymbol{F}})-\Psi^{\prime}(\boldsymbol{F}) \geq\left[\left(\frac{\partial \varphi}{\partial i_{1}}+i_{1} \frac{\partial \varphi}{\partial i_{2}}\right) \boldsymbol{R}-\frac{\partial \varphi}{\partial i_{2}} \boldsymbol{F}+\frac{\partial \varphi}{\partial i_{3}} \boldsymbol{F}^{*}\right] \cdot \boldsymbol{a} \otimes \boldsymbol{b} .
$$

Consider $\boldsymbol{a}$ and $\boldsymbol{b}$ such that $\boldsymbol{F}^{-1} \boldsymbol{a} \cdot \boldsymbol{b}=0$. For this choice (A2) reduces to

$$
\Psi^{\prime}(\overline{\boldsymbol{F}})-\Psi^{\prime}(\boldsymbol{F}) \geq\left(\frac{\partial \varphi}{\partial i_{1}}+i_{1} \frac{\partial \varphi}{\partial i_{2}}\right) \boldsymbol{a} \cdot \boldsymbol{R} \boldsymbol{b}-\frac{\partial \varphi}{\partial i_{2}} \boldsymbol{a} \cdot \boldsymbol{F} \boldsymbol{b}, \quad \text { with } J_{\bar{F}}=J_{F} .
$$

If $\boldsymbol{b}$ is an eigenvector of $\boldsymbol{U}$ with eigenvalue $\lambda$, then we have the further reduction

$$
\Psi^{\prime}(\overline{\boldsymbol{F}})-\Psi^{\prime}(\boldsymbol{F}) \geq\left[\frac{\partial \varphi}{\partial i_{1}}+\left(i_{1}-\lambda\right) \frac{\partial \varphi}{\partial i_{2}}\right] \boldsymbol{a} \cdot \boldsymbol{R} \boldsymbol{b} .
$$

Because $\boldsymbol{U}$ and $\boldsymbol{U}^{*}$ have the same eigenvectors, the restrictions on $\boldsymbol{a}$ and $\boldsymbol{b}$ imply that $\boldsymbol{a} \cdot \boldsymbol{R} \boldsymbol{b}=0$, yielding

$$
\Psi^{\prime}(\overline{\boldsymbol{F}})-\Psi^{\prime}(\boldsymbol{F}) \geq 0, \quad \text { with } J_{\bar{F}}=J_{F} .
$$

To interpret this result in the present context, we use $\overline{\boldsymbol{F}}-\boldsymbol{F}=\boldsymbol{a} \otimes \boldsymbol{b}$, with $\boldsymbol{F}^{-1} \boldsymbol{a} \cdot \boldsymbol{b}=0$, in the convexity conditions (46), obtaining $\overline{\boldsymbol{F}}^{*}-\boldsymbol{F}^{*}=-\boldsymbol{F}^{*} \boldsymbol{b} \otimes \boldsymbol{F}^{-1} \boldsymbol{a}$ and

$$
i_{1}(\overline{\boldsymbol{F}})-i_{1}(\boldsymbol{F}) \geq \boldsymbol{a} \cdot \boldsymbol{R}(\boldsymbol{F}) \boldsymbol{b} \quad \text { and } \quad i_{2}\left(\overline{\boldsymbol{F}}^{*}\right)-i_{2}\left(\boldsymbol{F}^{*}\right) \geq-\boldsymbol{U}^{*} \boldsymbol{b} \cdot \boldsymbol{F}^{-1} \boldsymbol{a},
$$

where the rotation invariance of the inner product has been used in the second inequality. The right-hand sides of these inequalities vanish for the choice of $\boldsymbol{b}$ leading to (A5). Thus, (A5) is equivalent to the restriction

$$
\varphi\left(\bar{\imath}_{1}, \bar{\imath}_{2}, i_{3}\right) \geq \varphi\left(i_{1}, i_{2}, i_{3}\right) \quad \text { for all } \bar{l}_{1} \geq i_{1} \text { and } \bar{\imath}_{2} \geq i_{2} .
$$

We observe that the restrictions $\bar{l}_{1} \geq i_{1}$ and $\bar{l}_{2} \geq i_{2}$ are satisfied in the formulas (73) pertaining to the free-swelling problem. Consequently the optimality of the solution (65) to that problem is a consequence of rank-one convexity, which of course is weaker than polyconvexity.

In a general deformation, the stretch invariants satisfy the restrictions $i_{1}^{2} \geq 3 i_{2}$ and $i_{2}^{2} \geq 3 i_{1} i_{3}$ [Podio-Guidugli and Vergara-Caffarelli 1991], with equality if and only if $\boldsymbol{U}$ is purely spherical. These impose the limits

$$
\sqrt{3 i_{2}} \leq i_{1} \leq \frac{i_{2}^{2}}{3 i_{3}} \quad \text { and } \quad \sqrt{3 i_{1} i_{3}} \leq i_{2} \leq \frac{i_{1}^{2}}{3}
$$

on the invariants; the first applying when $i_{2}$ and $i_{3}$ are specified and the second when $i_{1}$ and $i_{3}$ are specified. Fixing $\bar{l}_{2}=i_{2}$ and choosing $\bar{l}_{1}>i_{1}$ in the first interval, 
we write (A7) in the form

$$
\epsilon\left(\frac{\partial \varphi}{\partial i_{1}}+\epsilon^{-1} o(\epsilon)\right) \geq 0, \quad \text { with } \epsilon=\bar{\imath}_{1}-i_{1},
$$

which yields $(45)_{1}$ on dividing by $\epsilon(>0)$ and passing to the limit. In the same way (A7) is seen to imply (45) 2 . Thus the necessity of (45) for polyconvexity has been demonstrated. A discussion of necessary and sufficient conditions for polyconvexity in isotropic elasticity in terms of principal stretches is given in [Mielke 2005]. The idea for the present proof of necessity may be found in [Steigmann and Pipkin 1988], where it is applied to a special class of materials.

We note that if the free-energy function is strictly polyconvex, then the strict inequalities (44) and (45) follow. Moreover, if inequality (44) is strict and at least one of inequalities (45) is strict, then the free-energy function is strictly polyconvex. These statements are valid despite the fact that inequalities (46) are nonstrict [Steigmann 2003]. Finally, all of the foregoing remarks remain valid in the presence of a constraint on $J_{F}$, exemplified here by the swelling constraint.

\section{References}

[Adkins 1964] J. E. Adkins, "Non-linear diffusion, III: Diffusion through isotropic highly elastic solids", Philos. Trans. Roy. Soc. London Ser. A 256:1071 (1964), 301-316.

[Baek and Srinivasa 2004] S. Baek and A. R. Srinivasa, "Diffusion of a fluid through an elastic solid undergoing large deformations", Int. J. Non-Linear Mech. 39:2 (2004), 201-218.

[Ball 1977] J. M. Ball, "Convexity conditions and existence theorems in nonlinear elasticity", Arch. Rational Mech. Anal. 63:4 (1977), 337-403.

[Carroll 1988] M. M. Carroll, "Finite strain solutions in compressible isotropic elasticity", J. Elasticity 20:1 (1988), 65-92.

[Chester and Anand 2011] S. A. Chester and L. Anand, "A thermo-mechanically coupled theory for fluid permeation in elastomeric materials: application to thermally responsive gels", J. Mech. Phys. Solids 59:10 (2011), 1978-2006.

[Deng and Pence 2010] H. Deng and T. J. Pence, "Shear induced loss of saturation and in a fluid infused swollen hyperelastic cylinder”, Int J. Engng. Sci. 48:6 (2010), 624-646.

[Duda et al. 2010] F. P. Duda, A. C. Souza, and E. Fried, "A theory for species migration in a finitely strained solid with application to polymer network swelling”, J. Mech. Phys. Solids 58:4 (2010), $515-529$.

[Duda et al. 2011] F. P. Duda, A. C. Souza, and E. Fried, "Solvent uptake and cavitation", J. Mech. Phys. Solids 59:11 (2011), 2341-2354.

[Gurtin 2000] M. E. Gurtin, Configurational forces as basic concepts of continuum physics, Applied Mathematical Sciences 137, Springer, New York, 2000.

[Gurtin et al. 2010] M. E. Gurtin, E. Fried, and L. Anand, The mechanics and thermodynamics of continua, Cambridge University Press, 2010.

[Hong et al. 2008] W. Hong, X. Zhao, J. Zhou, and Z. Suo, "A theory of coupled diffusion and large deformation in polymeric gels", J. Mech. Phys. Solids 56:5 (2008), 1779-1793. 
[Liu 2002] I.-S. Liu, Continuum mechanics, Springer, Berlin, 2002.

[Mielke 2005] A. Mielke, "Necessary and sufficient conditions for polyconvexity of isotropic functions", J. Convex Anal. 12:2 (2005), 291-314.

[Neff 2003] P. Neff, "Some results concerning the mathematical treatment of finite multiplicative elasto-plasticity", pp. 251-274 in Deformation and failure in metallic and granular structures, edited by K. Hutter and H. Baaser, Lecture Notes in Applied and Computational Mech. 10, Springer, Berlin, 2003.

[Noll 1967] W. Noll, "Materially uniform simple bodies with inhomogeneities", Arch. Rational Mech. Anal. 27:1 (1967), 1-32.

[Noll 1970] W. Noll, "Representations of certain isotropic tensor functions", Arch. Math. (Basel) 21:1 (1970), 87-90.

[Ogden 1984] R. W. Ogden, Non-linear elastic deformations, Ellis Horwood, Chichester, 1984. Reprinted Dover, New York, 1997.

[Pence and Tsai 2005] T. J. Pence and H. Tsai, "On the cavitation of a swollen compressible sphere in finite elasticity", Int. J. Non-Linear Mech. 40:2-3 (2005), 307-321.

[Pence and Tsai 2006] T. J. Pence and H. Tsai, "Swelling-induced cavitation of elastic spheres", Math. Mech. Solids 11:5 (2006), 527-551.

[Podio-Guidugli 1991] P. Podio-Guidugli, "Polyconvex energies and symmetry requirements", $J$. Elasticity 26:3 (1991), 223-237.

[Podio-Guidugli and Vergara-Caffarelli 1991] P. Podio-Guidugli and G. Vergara-Caffarelli, "Extreme elastic deformations", Arch. Rational Mech. Anal. 115:4 (1991), 311-328.

[Prasad and Rajagopal 2006] S. C. Prasad and K. R. Rajagopal, "On the diffusion of fluids through solids undergoing large deformations”, Math. Mech. Solids 11:3 (2006), 291-305.

[Rajagopal 2003] K. R. Rajagopal, "Diffusion through polymeric solids undergoing large deformations”, Mat. Sci. Technol. 19:9 (2003), 1175-1180.

[Rajagopal and Srinivasa 2012] K. R. Rajagopal and A. Srinivasa, "Restrictions placed on constitutive relations by angular momentum balance and Galilean invariance", Z. Angew. Math. Phys. (2012).

[Rivlin 1977] R. S. Rivlin, "Some research directions in finite elasticity theory", Rheol. Acta 16:2 (1977), 101-112. Reprinted as pp. 418-429 of his Collected papers, vol. 1, edited by G. I. Barenblatt and D. D. Joseph, Springer, Berlin, 1997.

[Shi et al. 1981] J. J. Shi, K. R. Rajagopal, and A. S. Wineman, "Application of the theory of interacting continua to the diffusion of a fluid through a nonlinear elastic medium", Int. J. Engng. Sci. 19:6 (1981), 871-889.

[Steigmann 2002] D. J. Steigmann, "Invariants of the stretch tensors and their application to finite elasticity theory", Math. Mech. Solids 7:4 (2002), 393-404.

[Steigmann 2003] D. J. Steigmann, "On isotropic, frame-invariant, polyconvex strain-energy functions", Quart. J. Mech. Appl. Math. 56:4 (2003), 483-491.

[Steigmann and Pipkin 1988] D. J. Steigmann and A. C. Pipkin, "Stability of harmonic materials in plane strain", Quart. Appl. Math. 46:3 (1988), 559-568.

[Treloar 1975] L. R. G. Treloar, The physics of rubber elasticity, 3rd ed., Oxford University Press, 1975.

[Truesdell 1962] C. Truesdell, "Mechanical basis of diffusion", J. Chem. Phys. 37:10 (1962), 23362344. 
[Truesdell 1977] C. A. Truesdell, A first course in rational continuum mechanics, 1: General concepts, Academic Press, New York, 1977.

[Weitsman 1987] Y. Weitsman, "Stress assisted diffusion in elastic and viscoelastic materials", $J$. Mech. Phys. Solids 35:1 (1987), 73-94.

Received 8 May 2012. Revised 25 Jul 2012. Accepted 26 Sep 2012.

GARY J. TEMPLET: gjtempl@berkeley.edu

Department of Mechanical Engineering, University of California, Berkeley, Berkeley, CA 94720,

United States

DAVID J. STEIGMANN: steigman@me berkeley.edu

Department of Mechanical Engineering, University of California, Berkeley, Berkeley, CA 94720,

United States 
EDITORIAL BOARD

ANTONIO CARCATERRA ERIC A. CARLEN

FRANCESCO DELL' ISOLA

RAFFAELE ESPOSITO

ALBERT FANNJIANG

Gilles A. FrancForT

PiERANGelo MARCATI

JEAN-JACQUES MARIGo

PETER A. MARKOWICH

MARTIN OSTOJA-STARZEWSKI

PIERRE SEPPECHER

David J. SteigmanN

Paul STEINMANN

Pierre M. Suquet

\section{MANAGING EDITORS}

MICOL AMAR

CORRADO LATTANZIO

ANGELA MADEO

MARTIN OSTOJA-STARZEWSKI

\section{ADVISORY BOARD}

ADNAN AKAY

Holm AltenbaCH

MICOL AMAR

HARM ASKES

TEODOR ATANACKOVIĆ

VICTOR BERDICHEVSKY

GuY BouchitTÉ

ANDREA BRAIDES

ROBERTO CAMASSA

ERIC DARVE

Felix Darve

ANNA DE Masi

Gianpietro Del Piero

EMmanuele Di BenedetTo BERNOLD FIEDLER

IRENE M. GAMBA

SERGEy GavriLyUK

Timothy J. HEALEY

DOMINIQUE JEULIN ROGER E. KHAYAT

CorRado LATTANZIO ROBERT P. LIPTON

ANGELO LUONGO

ANGELA MADEO

JUAN J. MANFREDI

CARLO MARCHIORO

GÉrard A. Maugin

ROBERTO NATALINI

PATRIZIO NEFF

ANDREY PIATNITSKI

ERRICO PRESUTTI

MARIO PUlVIRENTI

LuCIO RusSO

Miguel A. F. SAnjuan

PATRICK SElVAdURA

ALEXANDER P. SEYRANIAN

MiroslaV ŠILHAVÝ

GUIDO SWEERS

ANTOINETTE TORDESILLAS

LEV TRUSKINOVSKY

JUAN J. L. VELÁZQUEZ

VINCENZO VESPRI

ANGELO VULPIANI msp.org/memocs

Università di Roma “La Sapienza”, Italia

Rutgers University, USA

(CO-CHAIR) Università di Roma "La Sapienza", Italia

(TREASURER) Università dell'Aquila, Italia

University of California at Davis, USA

(CO-CHAIR) Université Paris-Nord, France

Università dell'Aquila, Italy

École Polytechnique, France

DAMTP Cambridge, UK, and University of Vienna, Austria

(CHAIR MANAGING EDITOR) Univ. of Illinois at Urbana-Champaign, USA

Université du Sud Toulon-Var, France

University of California at Berkeley, USA

Universität Erlangen-Nürnberg, Germany

LMA CNRS Marseille, France

Università di Roma "La Sapienza”, Italia

Università dell' Aquila, Italy

Université de Lyon-INSA (Institut National des Sciences Appliquées), France (CHAIR MANAGING EDITOR) Univ. of Illinois at Urbana-Champaign, USA

Carnegie Mellon University, USA, and Bilkent University, Turkey

Otto-von-Guericke-Universität Magdeburg, Germany

Università di Roma "La Sapienza", Italia

University of Sheffield, UK

University of Novi Sad, Serbia

Wayne State University, USA

Université du Sud Toulon-Var, France

Università di Roma Tor Vergata, Italia

University of North Carolina at Chapel Hill, USA

Stanford University, USA

Institut Polytechnique de Grenoble, France

Università dell'Aquila, Italia

Università di Ferrara and International Research Center MEMOCS, Italia

Vanderbilt University, USA

Freie Universität Berlin, Germany

University of Texas at Austin, USA

Université Aix-Marseille, France

Cornell University, USA

École des Mines, France

University of Western Ontario, Canada

Università dell' Aquila, Italy

Louisiana State University, USA

Università dell' Aquila, Italia

Université de Lyon-INSA (Institut National des Sciences Appliquées), France

University of Pittsburgh, USA

Università di Roma "La Sapienza", Italia

Université Paris VI, France

Istituto per le Applicazioni del Calcolo "M. Picone", Italy

Universität Duisburg-Essen, Germany

Narvik University College, Norway, Russia

Università di Roma Tor Vergata, Italy

Università di Roma “La Sapienza”, Italia

Università di Roma "Tor Vergata", Italia

Universidad Rey Juan Carlos, Madrid, Spain

McGill University, Canada

Moscow State Lomonosov University, Russia

Academy of Sciences of the Czech Republic

Universität zu Köln, Germany

University of Melbourne, Australia

École Polytechnique, France

Bonn University, Germany

Università di Firenze, Italia

Università di Roma La Sapienza, Italia

MEMOCS (ISSN 2325-3444 electronic, 2326-7186 printed) is a journal of the International Research Center for the Mathematics and Mechanics of Complex Systems at the Università dell'Aquila, Italy.

Cover image: "Tangle” by @ John Horigan; produced using the Context Free program (contextfreeart.org).

\section{PUBLISHED BY}

\section{7 mathematical sciences publishers nonprofit scientific publishing}

http://msp.org/

(C) 2013 Mathematical Sciences Publishers 
Mathematics and Mechanics of Complex Systems vol. 1 no. 1

Dislocations, imperfect interfaces and interface cracks in anisotropic elasticity for quasicrystals

Xu Wang and Peter Schiavone

Localization of point vortices under curvature perturbations

Roberto Garra

Contraction of the proximal map and generalized convexity of the Moreau-Yosida regularization in the 2-Wasserstein metric

Eric A. Carlen and Katy Craig

Ptolemy's longitudes and Eratosthenes' measurement of the earth's circumference

\section{Lucio Russo}

TV-min and greedy pursuit for constrained joint sparsity and application to inverse scattering

Albert Fannjiang

On the theory of diffusion and swelling in finitely deforming elastomers

Gary J. Templet and David J. Steigmann

MEMOCS is a journal of the International Research Center for the Mathematics and Mechanics of Complex Systems at the Università dell' Aquila, Italy.

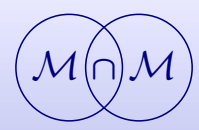

